\title{
Smartphones and e-tablets in perioperative medicine
}

\author{
Frederic Michard \\ MiCo Sàrl, Denens, Switzerland
}

Smartphones and electronic tablets (e-tablets) have become ubiquitous devices. Their ease of use, smartness, accessibility, mobility and connectivity create unique opportunities to improve quality of surgical care from prehabilitation to rehabilitation. Before surgery, digital applications (Apps), serious games and text messaging may help for a better control of risk factors (hypertension, overweight), for smoking cessation, and for optimizing adherence to preoperative recommendations (e.g., regarding anticoagulation or antihypertensive treatments). During surgery, Apps may help to rationalize fluid management and estimate blood loss. After surgery, smartphones and/or connected sensors (pulse oximeter, adhesive path, electronic tattoo, bioimpedance necklace) can be used to monitor body temperature, heart rate, heart rate variability (detection of cardiac arrhythmia), respiratory rate, arterial oxygen saturation and thoracic fluid content. Therefore, these tools have potential for the early detection of infectious, cardiac and respiratory complications in the wards and from home. When connected to echo probes, smartphones and e-tablets can also be used as ultrasound devices during central venous catheter insertion, for peripheral nerve blocks, and to perform echocardiography in patients developing cardiac complications. Finally, electronic checklists now exist as Apps to enhance communication between patients and healthcare professionals, and to track and record step by step each element of the surgical journey. Studies are now urgently needed to investigate whether this digital revolution can translate into a better outcome, an earlier detection of postoperative complications, a decrease in hospital readmissions and in health care costs.

Key Words: Digital application, Electronic tablet, Perioperative medicine, Smartphone, Surgical complication, Wearable sensor.

\section{Introduction}

There are over 320 million inpatient surgical procedures worldwide [1]. We learn from the recent International Surgical Outcome Study [2] that around $17 \%$ of these patients develop one or more complications, and among them, that $2.8 \%$ die from their complications. One can therefore estimate that around 1.5 million patients/yr (or 3 patients/min!) die of post-

Corresponding author: Frederic Michard, M.D., Ph.D.

MiCo Sàrl, Chemin de Chapallaz 4, 1135 Denens, Switzerland

Tel: 41-021-803-3004, Fax: 41-79-599-09-6

Email: frederic.michard@bluewin.ch, michardconsulting.com

Received: August 24, 2017.

Accepted: August 29, 2017.

Korean J Anesthesiol 2017 October 70(5): 493-499

https://doi.org/10.4097/kjae.2017.70.5.493 operative complications. In the United States (US), if postoperative mortality was part of the official statistics from the Centers for Disease Control and Prevention, it would represent the third leading cause of death, right after cancer [3]. Postoperative complications are not only a human burden. They also dramatically increase hospital costs [4].

Several initiatives have been developed to improve quality of surgical care, from the WHO surgical safety checklist, to minimally invasive surgery, protective mechanical ventilation, and enhanced recovery programs. Smartphones and electronic tablets (e-tablets) are now ubiquitous devices. Their ease of use, smartness, accessibility, mobility and connectivity create unique opportunities to transform medicine and improve quality of care [5]. Potential clinical applications of smartphones and etablets in surgical patients will be described and discussed in this manuscript, from prehabilitation to rehabilitation.

(c) This is an open-access article distributed under the terms of the Creative Commons Attribution Non-Commercial License (http://creativecommons.org/ licenses/by-nc/4.0/), which permits unrestricted non-commercial use, distribution, and reproduction in any medium, provided the original work is properly cited. 


\section{Prehabilitation}

Prehabilitation is known to have an impact on postoperative outcome. Preoperative change in physical status and a better control of risk factors can be facilitated by digital tools and applications (Apps) downloaded on smartphones or e-tablets. Connected devices such as wireless brachial cuffs and electronic scales can be used for self-monitoring of blood pressure and weight, and the visualization of trends over time. Apps, text messaging and serious games (video games designed for training and education) have been shown to help for a better control of hypertension, to reduce overweight, as well as for smoking cessation [6,7]. Multiple activity trackers and Apps now exist to invite patients monitor and increase their physical activity. These digital tools seem to be effective only for a short period of time [8], which is clearly an issue when dealing with chronic conditions and diseases, but less of a problem if used during the weeks preceding surgery (Fig. 1). Electronic checklists are Apps developed to optimize communication between patients and healthcare professionals during the entire surgical journey. Before surgery they can be used to ensure patients follow preoperative recommendations, in particular regarding medications (Fig. 2).

\section{Intraoperative Management}

\section{Point of care echography for regional anesthesia and central venous catheter insertion}

Two-dimensional ultrasound offer gains in safety and quality during central venous catheter insertion when compared with anatomical landmark techniques [9]. There is also evidence that

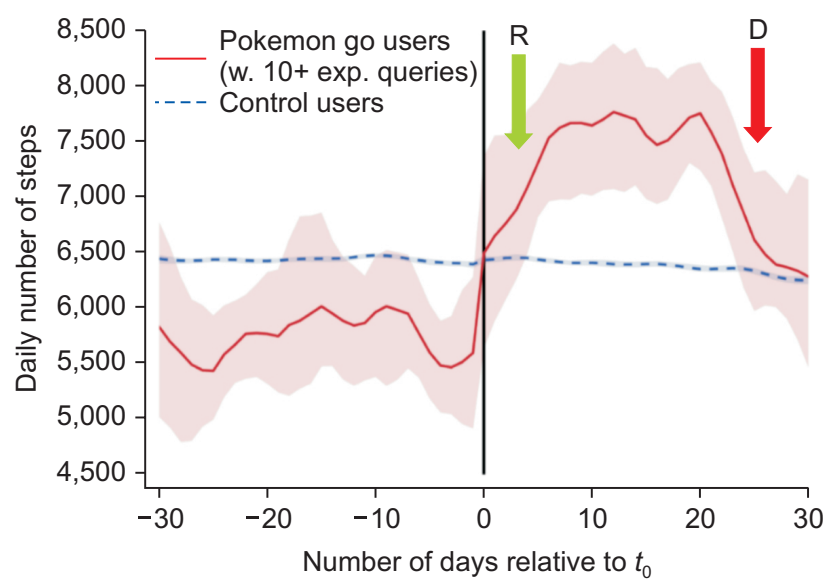

Fig. 1. Potential impact of an electronic game on physical activity. $\mathrm{R}=$ rise in physical activity in game users vs controls, $\mathrm{D}=$ dropout effect observed after 3 weeks. Adapted from (8) with permission. peripheral nerve blocks performed by ultrasound guidance are superior in terms of improved sensory and motor block, reduced need for supplementation and fewer minor complications [10]. Ultrasound machines dramatically evolved over time from bulky and heavy devices to pocket tools. Echo probes can now be directly connected to a smartphone or an e-tablet (Fig. 3) and may soon replace stethoscopes in anesthesiologists' pockets.

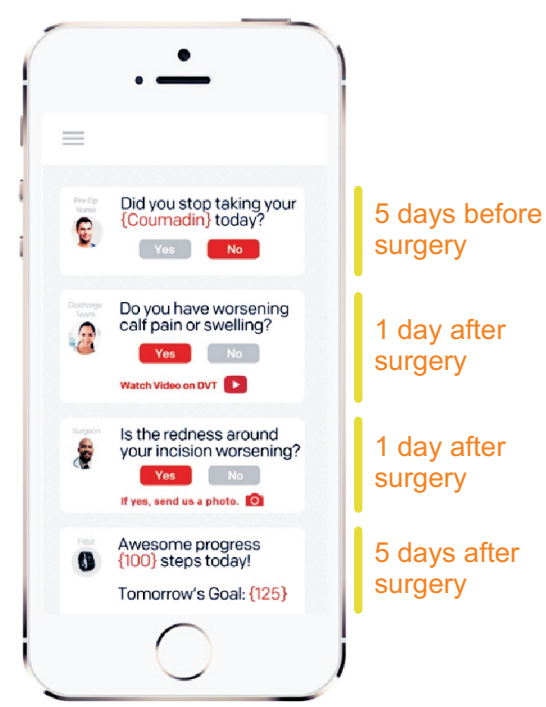

Fig. 2. Example of electronic checklist. This App is designed to optimize communication between patients and healthcare professionals, to improve the adherence to and record all the elements of the surgical journey. From Twistle.com.

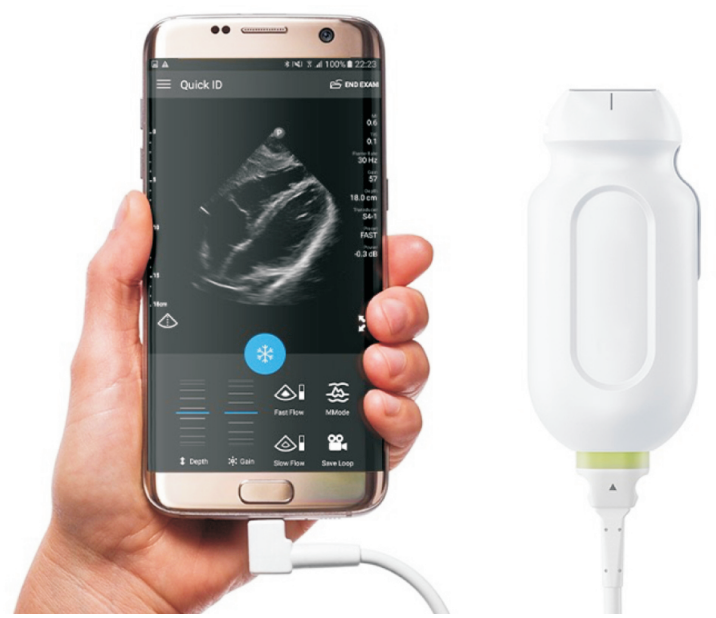

Fig. 3. Example of point of care (POC) ultrasound system. Before surgery, the echo probe, connected to a smartphone or an e-tablet, can be used to guide regional anesthesia and central venous catheter insertion. In the surgical wards, it can be used for echocardiography in case of cardio-respiratory deterioration. Echo probe from Philips.com. 


\section{Fluid management}

Intraoperative fluid management is a key determinant of postoperative outcome. Fluid overload has been known for a while as responsible for complications related to tissue edema (e.g., anastomotic leak, prolonged mechanical ventilation), so that fluid restriction has been encouraged at some point. However, recent studies have clearly demonstrated that insufficient fluid administration is also associated with a significant increase in postoperative complications. Therefore, titrating or tailoring fluid administration to individual needs is highly desirable to ensure patients receive the right amount of fluid at the right time [11]. Preventing unjustified fluid administration by detecting fluid unresponsiveness has been shown to be useful to decrease postoperative morbidity, hospital length of stay and costs [12]. Dynamic variables such as pulse pressure variation (PPV) are useful to detect fluid unresponsiveness and are now displayed on most but not all bedside monitors. Therefore, an App has been developed to quantify PPV from the picture of the arterial pressure waveform taken with a smartphone (Fig. 4). A simulation study showed it works reasonably well if 3 pictures and PPV estimations are performed and averaged [13]. This App has potential to compute stroke volume and cardiac output from the arterial pressure waveform [14], the accuracy and precision depending on the pulse contour algorithm that will be used by the App.

\section{Blood management}

The quantification of surgical blood loss may be challenging and is often inaccurate. An App has been developed to quantify more objectively blood loss in surgical sponges and suction

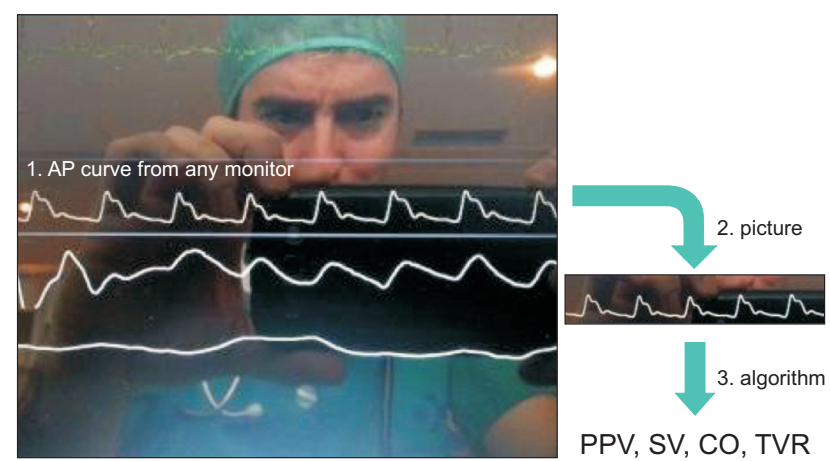

Fig. 4. Example of App for hemodynamic monitoring. This App computes advanced hemodynamic parameters from an arterial pressure (AP) waveform (1). A picture of the AP curve is taken by the user (2), then the App calculates the pulse pressure variation (PPV) and has potential to compute stroke volume (SV), cardiac output (CO) and total vascular resistance (TVR) from any pulse contour algorithm (3). From Capstesia. com. canisters. The App analyzes a picture taken by an e-tablet to estimate the hemoglobin mass present on the sponges or in the canister and extrapolate the estimated blood loss (Fig. 5). Recent studies suggest that the App is more accurate than the gravimetric method and more precise than the visual estimation done by clinicians $[15,16]$. It may help to rationalize blood transfusion and decrease associated complications.

\section{Postoperative Management}

Recent studies have shown that around one third of deaths after surgery occur in the wards [2]. This situation has been called «failure to rescue » and has been proposed to explain why hospitals with comparable postoperative morbidity rates may have very different mortality rates: outcome depends more on the ability to detect early and treat properly postoperative complications than in the occurrence of complications [17]. Importantly, studies have also shown that most patients start deteriorating hours before medical teams are called for rescue or intensive care unit (ICU) transfer. In this regard, several studies have logically demonstrated the value of continuous monitoring systems for the early detection of deteriorating patients. For instance, the use of pulse oximeters to continuously monitor arterial oxygen saturation $\left(\mathrm{SpO}_{2}\right)$ and heart rate in 2,841 orthopedic patients (many of them receiving opioids) was associated with a significant decrease in the number of rescue events and ICU transfers [18]. The use of a piezoelectric contact-free sensor (placed under the mattress) to continuously monitor heart rate and respiratory rate in 2,314 medico-surgical patients was associated with a significant decrease in the number of calls for cardiac arrest and hospital length of stay [19]. More recently, the

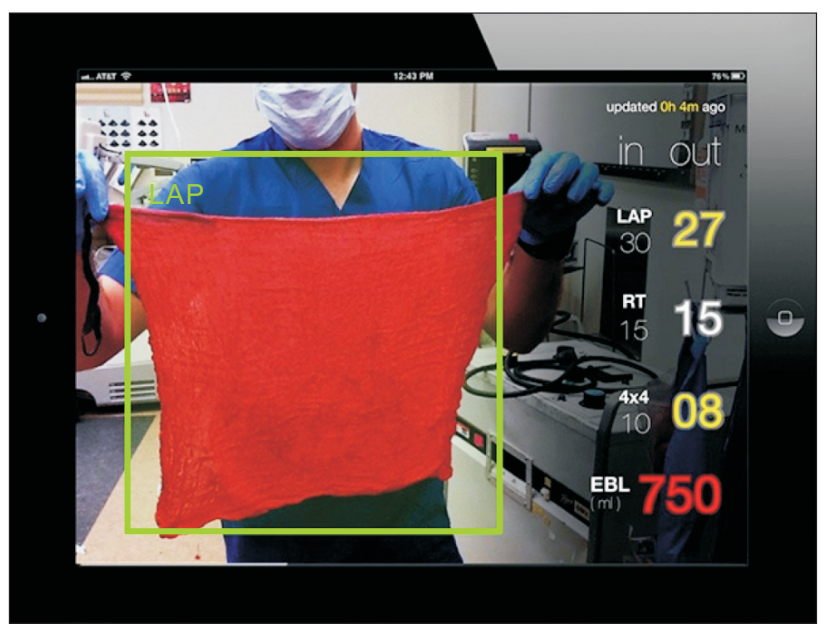

Fig. 5. Example of App designed to quantify surgical blood loss. A picture of the surgical sponge (LAP) is taken by the user, then the App estimates the hemoglobin loss and extrapolate the surgical blood loss. $\mathrm{EBL}=$ cumulative estimated blood loss. From Gausssurgical.com. 
use of wireless sensors to monitor vital signs $\left(\mathrm{SpO}_{2}\right.$, heart rate, blood pressure, respiratory rate), automatically calculate an early warning score, and alert nurses in case of deterioration, was associated with a significant decrease in the number of cardiac arrests and in mortality [20].

Multiple sensors are becoming available for proactive and wireless monitoring in ambulatory patients [21,22]. Thanks to stretchable and flexible electronics, electronic tattoos will soon be available [23]. Technically speaking, physiologic signals can be transmitted to, processed by, and displayed on smartphones or e-tablets (Fig. 6). Smart algorithms and software are currently under development to filter artifacts in ambulatory patients and prevent alarm fatigue, as well as to fuse multiple vital signs into a single wellness index or warning score. Such indexes or scores are susceptible to improve the detection of clinical deterioration at an early stage and should help to tackle failure to rescue $[21,23]$. Once health care professionals have been alerted, pocket echo devices or probes (Fig. 3) can be useful to provide information about cardiac function and clarify the underlying mechanisms of clinical deterioration.

\section{Rehabilitation}

\section{Activity trackers}

Early mobilization is a key element of postoperative recovery.
Multiple wrist, waist or ankle sensors with accelerometers are now available to detect movements and quantify physical activity. They are usually wirelessly connected to smartphones and e-tablets to display, track and exchange the information. Some have been used with success for the objective assessment of early mobilization after surgery [24-26]. However, it is important to keep in mind that activity trackers may have limitations during low speed exercises, which is often the case immediately after surgery [27].

\section{Home monitoring}

Smartphones and connected devices have potential value to diagnose and better characterize postoperative complications occurring after hospital discharge. From the phone itself it is possible to measure heart rate and respiratory rate. Heart rate, or actually pulse rate, can be derived from a peripheral pulse signal obtained by putting a finger on the camera of the cell phone. The App uses the flash light, as a pulse oximeter would use red and infrared lights, to detect the finger pulse. Studies have shown that some of the Apps are accurate to measure pulse rate and to detect abnormal cardiac rhythms [28,29]. Respiratory rate can also be estimated from the respiratory oscillations of the pulse signal [30], from the video recording of chest and abdomen movements [31], or from the nasal breath sound recording with the smartphone microphone [32].
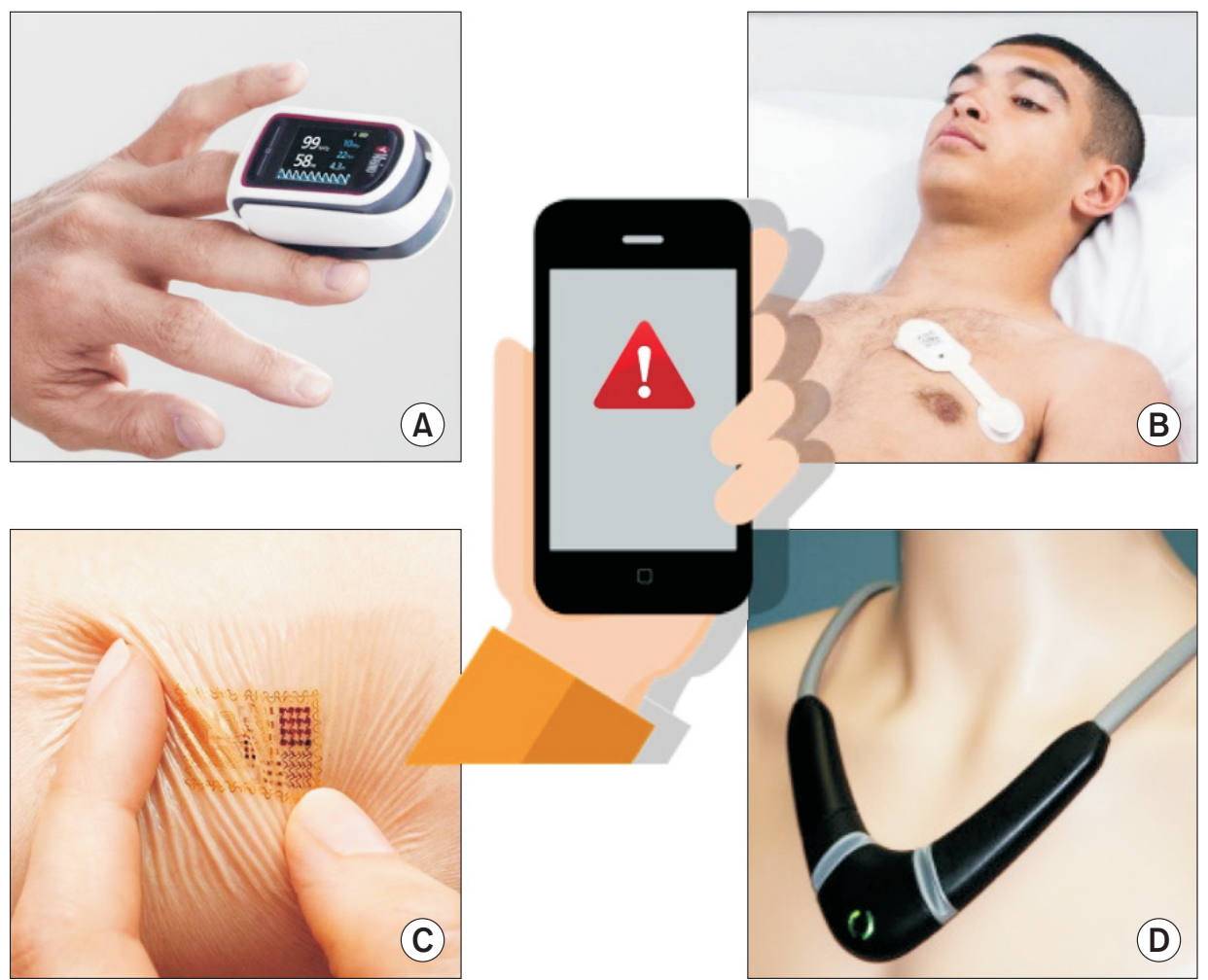

(C)

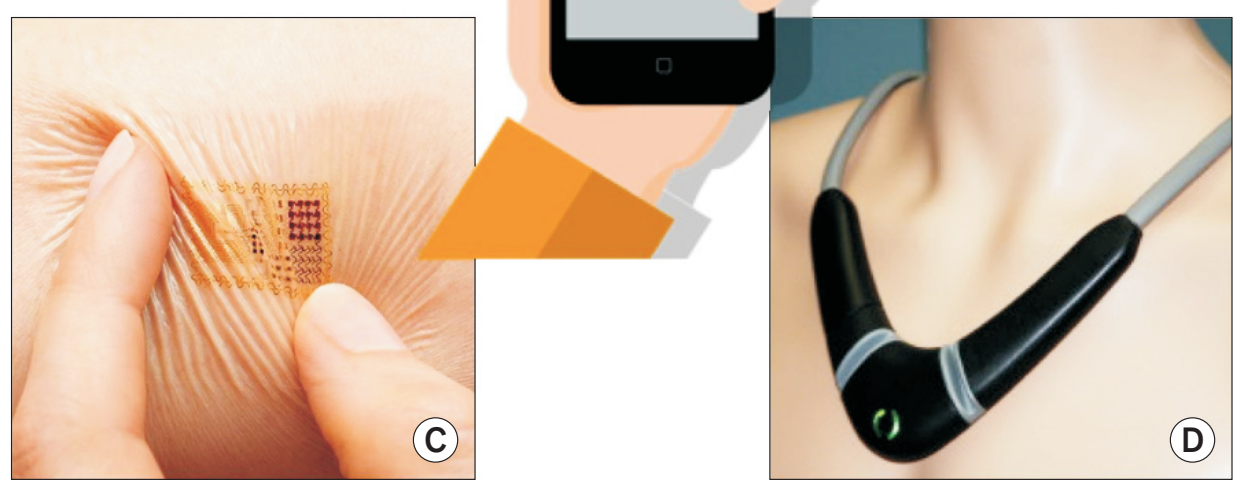

Fig. 6. Example of mobile monitoring systems for ambulatory patients and/ or home monitoring. $\mathrm{A}=$ pulse oximeter from Masimo.com, $\mathrm{B}=$ adhesive patch from Isansys.com, $\mathrm{C}=$ electronic tattoo from mc10inc.com, $\mathrm{D}=$ Necklace from Tosense.com. The information is transmitted to a mobile display (e.g. a smartphone) so that healthcare professionals and/or patients are informed early in case of clinical deterioration. 
Connected devices can be used to measure, store and transmit additional parameters. Medical grade wireless brachial cuffs and pulse oximeters are now available in consumer stores and can be used to accurately monitor blood pressure and arterial oxygen saturation. Electrocardiography (ECG) recordings are possible with the use of a special case equipped with electrodes (Fig. 7). This system is FDA approved and has been shown to be reliable to measure QT interval, detect atrial fibrillation and ST segment elevation [33]. Other wearable sensors (e.g., adhesive patch or necklace) are capable of measuring skin temperature or thoracic fluid content (Fig. 6). The formers are useful to detect infectious complications. The later are susceptible to detect pulmonary edema before patients develop any symptoms, which may be useful to trigger early intervention and prevent hospital readmission. Finally, the above mentioned electronic checklists can help to track and describe complications (Fig. 2). For example, surgical wound pictures can be taken and shared with health care professionals who can therefore provide appropriate and immediate guidance to the patients.

In summary, self and home monitoring of temperature, heart rate, heart rate variability (detection of arrhythmia), ECG leads, blood pressure, respiratory rate, arterial oxygen saturation, and thoracic fluid content is now a reality. Studies are urgently needed to investigate whether this digital revolution will translate into a better and earlier detection of postoperative complications and a decrease in hospital readmissions and health care costs.

\section{Novelty Blindness and Gizmo Idolatry}

Multiple Apps and connected devices have become available over the last few years and, given their ease of use and low cost, the temptation is to integrate them into clinical practice before they have been validated and/or have demonstrated clinical value. In other words, the main risk associated with the use of digital innovations and wearable devices is novelty blindness.

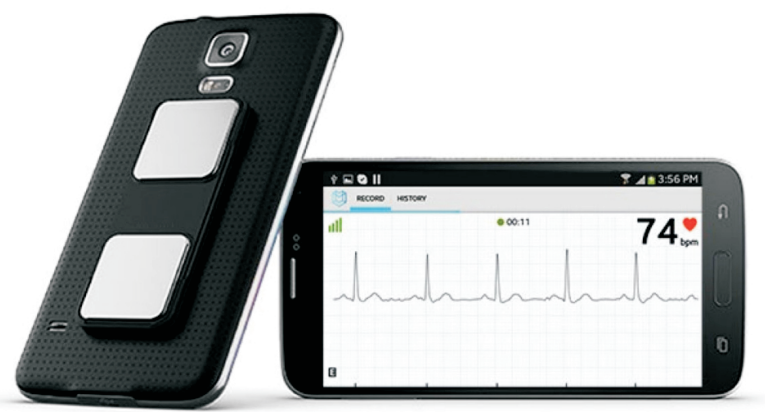

Fig. 7. Example of smartphone case for electrocardiography (ECG) recording. This case includes 2 electrodes. Putting fingers on the electrodes enables the recording of a single lead ECG that can be recorded and shared with healthcare professionals. From Alivecor.com.
The "Instant Blood Pressure" App was developed to measure blood pressure with a smartphone, from the simultaneous recording of a balistogram (smartphone on the chest detecting heart contraction from built-in accelerometers) and a peripheral pulse (finger on the camera). The time difference between the heart beat and the arrival of the corresponding peripheral pulse is the pulse wave transit time (PWTT). It is known to be inversely proportional to blood pressure. However, the relationship between PWTT and blood pressure is different from one patient to the other, and for a given patient, it is dependent on cardiac output, which may vary with physical activity and underlying diseases (e.g. cirrhosis, heart failure). In other words, it is unlikely that this method may be useful to measure accurate absolute blood pressure values without regular calibrations. Nevertheless, over 140,000 people had downloaded the App on their smartphone before it was removed from market after a publication from the Johns Hopkins hospital. This study showed it was not accurate and missed most hypertension cases [34]. The Scout device from Scanadu is another gadget that was supposed to measure blood pressure from a similar principle, by quantifying the time difference between a forehead pulse (device on the forehead) and a finger pulse (index on the device). Scanadu had been able to raise first $\$ 1.5 \mathrm{M}$ from crowdfunding, then $\$ 35 \mathrm{M}$ in series B funding, bringing a lot of attention around this new product. Recently, following the disappointing (but expected) results of a clinical evaluation, the company was forced by the FDA to withdraw the product and its App from the market [35]. These examples illustrate well the risk of gizmo idolatry [36]. If some new connected devices and Apps have value, many lack of reliability. Serious clinical evaluations are therefore needed before clinical implementation. Given their expertise in physiologic monitoring and clinical research, anesthesiologists are well positioned to lead this endeavor.

\section{Conclusion}

Postoperative complications are a major clinical and economic burden. Each year, they are responsible for over 1 million deaths. Many complications and deaths could be prevented with a better perioperative management. Smartphones and e-tablets are ubiquitous devices that can help both healthcare professionals and patients to better prepare for surgery, to improve intraoperative management and postoperative monitoring in the hospital and beyond. These tools may be especially useful in developing countries and small hospitals where expensive medical technologies are not always readily available. 


\section{Conflict of Interest}

Dr. Michard is the founder and managing director of MiCo, a
Swiss consulting firm providing services to medtech companies, digital health startups and life science investors.

\section{References}

1. Weiser TG, Haynes AB, Molina G, Lipsitz SR, Esquivel MM, Uribe-Leitz T, et al. Estimate of the global volume of surgery in 2012: an assessment supporting improved health outcomes. Lancet 2015; 385 (2 Suppl): S11.

2. International Surgical Outcomes Study group. Global patient outcomes after elective surgery: prospective cohort study in 27 low-, middleand high-income countries. Br J Anaesth 2016; 117: 601-9.

3. Bartels K, Karhausen J, Clambey ET, Grenz A, Eltzschig HK. Perioperative organ injury. Anesthesiology 2013; 119: 1474-89.

4. Michard F, Mountford WK, Krukas MR, Ernst FR, Fogel SL. Potential return on investment for implementation of perioperative goaldirected fluid therapy in major surgery: a nationwide database study. Perioper Med (Lond) 2015; 4: 11.

5. Lee JH. Future of the smartphone for patients and healthcare providers. Healthc Inform Res 2016; 22: 1-2.

6. Stephens J, Allen J. Mobile phone interventions to increase physical activity and reduce weight: a systematic review. J Cardiovasc Nurs 2013; 28: 320-9.

7. Whittaker R, McRobbie H, Bullen C, Rodgers A, Gu Y. Mobile phone-based interventions for smoking cessation. Cochrane Database Syst Rev 2016; 4: CD006611.

8. Althoff T, White RW, Horvitz E. Influence of pokémon go on physical activity: study and implications. J Med Internet Res 2016 ; 18 : e315.

9. Brass P, Hellmich M, Kolodziej L, Schick G, Smith AF. Ultrasound guidance versus anatomical landmarks for internal jugular vein catheterization. Cochrane Database Syst Rev 2015; 1: CD006962.

10. Lewis SR, Price A, Walker KJ, McGrattan K, Smith AF. Ultrasound guidance for upper and lower limb blocks. Cochrane Database Syst Rev 2015; (9): CD006459.

11. Michard F, Biais M. Rational fluid management: dissecting facts from fiction. Br J Anaesth 2012; 108: 369-71.

12. Benes J, Giglio M, Brienza N, Michard F. The effects of goal-directed fluid therapy based on dynamic parameters on post-surgical outcome: a meta-analysis of randomized controlled trials. Crit Care 2014; 18: 584.

13. Desebbe O, Joosten A, Suehiro K, Lahham S, Essiet M, Rinehart J, et al. A novel mobile phone application for pulse pressure variation monitoring based on feature extraction technology: a method comparison study in a simulated environment. Anesth Analg 2016; 123: 10513.

14. Barrachina B, Cobos R, Mardones N, Castañeda A, Vinuesa C. Assessment of a smartphone app (Capstesia) for measuring pulse pressure variation: agreement between two methods: A Cross-sectional study. Eur J Anaesthesiol 2017; 34: 75-80.

15. Holmes AA, Konig G, Ting V, Philip B, Puzio T, Satish S, et al. Clinical evaluation of a novel system for monitoring surgical hemoglobin loss. Anesth Analg 2014; 119: 588-94.

16. Konig G, Waters JH, Javidroozi M, Philip B, Ting V, Abbi G, et al. Real-time evaluation of an image analysis system for monitoring surgical hemoglobin loss. J Clin Monit Comput 2017 [Epub ahead of print].

17. Ghaferi AA, Birkmeyer JD, Dimick JB. Variation in hospital mortality associated with inpatient surgery. N Engl J Med 2009; 361: $1368-75$.

18. Taenzer AH, Pyke JB, McGrath SP, Blike GT. Impact of pulse oximetry surveillance on rescue events and intensive care unit transfers: a before-and-after concurrence study. Anesthesiology 2010; 112: 282-7.

19. Brown H, Terrence J, Vasquez P, Bates DW, Zimlichman E. Continuous monitoring in an inpatient medical-surgical unit: a controlled clinical trial. Am J Med 2014; 127: 226-32.

20. Subbe CP, Duller B, Bellomo R. Effect of an automated notification system for deteriorating ward patients on clinical outcomes. Crit Care 2017; 21: 52 .

21. Michard F, Gan TJ, Kehlet H. Digital innovations and emerging technologies for enhanced recovery programmes. Br J Anaesth 2017 [Epub ahead of print].

22. Ross ES, Sakakibara BM, Mackay MH, Whitehurst DG, Singer J, Toma M, et al. The use of text messaging to improve the hospital-tocommunity transition in acute coronary syndrome patients (Txt2Prevent): intervention development and pilot randomized controlled trial protocol. JMIR Res Protoc 2017; 6: e91.

23. Michard F. Hemodynamic monitoring in the era of digital health. Ann Intensive Care 2016; 6: 15.

24. Luna IE, Peterson B, Kehlet H, Aasvang EK. Individualized assessment of post-arthroplasty recovery by actigraphy: a methodology study. J Clin Monit Comput 2016 [Epub ahead of print].

25. Cook DJ, Thompson JE, Prinsen SK, Dearani JA, Deschamps C. Functional recovery in the elderly after major surgery: assessment of mobility recovery using wireless technology. Ann Thorac Surg 2013; 96: 1057-61.

26. Mobbs RJ, Phan K, Maharaj M, Rao PJ. Physical activity measured with accelerometer and self-rated disability in lumbar spine surgery: a prospective study. Global Spine J 2016; 6: 459-64. 
27. Beevi FH, Miranda J, Pedersen CF, Wagner S. An evaluation of commercial pedometers for monitoring slow walking speed populations. Telemed J E Health 2016; 22: 441-9.

28. Matsumura K, Yamakoshi T. iPhysioMeter: a new approach for measuring heart rate and normalized pulse volume using only a smartphone. Behav Res Methods 2013; 45: 1272-8.

29. Chan PH, Wong CK, Poh YC, Pun L, Leung WW, Wong YF, et al. Diagnostic performance of a smartphone-based photoplethysmographic application for atrial fibrillation screening in a primary care setting. J Am Heart Assoc 2016; 5. e003428.

30. Nam Y, Lee J, Chon KH. Respiratory rate estimation from the built-in cameras of smartphones and tablets. Ann Biomed Eng 2014; 42: 88598.

31. Nam Y, Kong Y, Reyes B, Reljin N, Chon KH. Monitoring of heart and breathing rates using dual cameras on a smartphone. PLoS One 2016; 11: e0151013.

32. Nam Y, Reyes BA, Chon KH. Estimation of respiratory rates using the built-in microphone of a smartphone or headset. IEEE J Biomed Health Inform 2016; 20: 1493-501.

33. Saxon LA. Ubiquitous wireless ECG recording: a powerful tool physicians should embrace. J Cardiovasc Electrophysiol 2013; 24: 480-3.

34. Plante TB, Urrea B, MacFarlane ZT, Blumenthal RS, Miller ER 3rd, Appel LJ, et al. Validation of the instant blood pressure smartphone app. JAMA Intern Med 2016; 176: 700-2.

35. Scanadu to shut down support for its Scout device per FDA regulation and customers are mad [updated 2016 Dec 13; cited 2017 Aug 22]. Avaliable from https://techcrunch.com/2016/12/13/fda-orders-scanadu-to-shut-down-support-for-its-scout-device-and-customers-are$\mathrm{mad} /$.

36. Leff B, Finucane TE. Gizmo idolatry. JAMA 2008; 299: 1830-2. 